\title{
Research of Water Response under the Action of the Infrared Human Body Radiation by Water Conductometric Sensors
}

\author{
Gennady G. Shishkin ${ }^{1}$, Igor M. Ageev, Yury M. Rybin', Alexei G. Shishkin ${ }^{2}$ \\ ${ }^{1}$ Moscow Aviation Institute, National Research University, Moscow, Russia \\ ${ }^{2}$ Moscow State University, Moscow, Russia \\ Email: shishkin@cs.msu.su
}

Received April 26, 2013; revised May 26, 2013; accepted June 2, 2013

Copyright (C) 2013 Gennady G. Shishkin et al. This is an open access article distributed under the Creative Commons Attribution License, which permits unrestricted use, distribution, and reproduction in any medium, provided the original work is properly cited.

\begin{abstract}
Non-equilibrium thermal and biothermal radiation generated by heated solid materials and hematothermal living organisms are studied by water conductometric sensors. Engineering aspects and physical features of developed water conductometric sensors are given. Procedure and measuring technique are described. Our experiments show the anomalous behavior of water conductivity and associated differential parameters under water heating by biological objects compared with traditional heating sources. Water response to human action strongly depends on psychophysiological and psychoemotional state of the person. Moreover the responses to the action by left and right human hands are substantially different and as a rule are specific to the gender. The possible physicochemical mechanisms of such anomalous water behavior are studied. It is suggested that the observed effects are associated with resonant excitation of vibration-rotation energy levels of water under the influence of bioradiation generated by human organism consisting of approximately $70 \%$ water. The results obtained have good perspectives for future applications in different fields of human activity.
\end{abstract}

Keywords: Water Electrical Conductivity; Conductometric Sensors; Infrared Human Body Radiation; Bioradiation

\section{Introduction}

At present there is a certain amount of experimental data demonstrating that many electrophysical properties of matter are dependent not only on temperature magnitude but on type of heating source as well [1-4]. This problem is connected with emissivity of heated bodies [1] that can vary within wide limits. It was shown [2] that day long water exposure to copper radiation at room temperature led to the changes of monochromatic coefficient of water transmittance within $6 \%-8 \%$ in the spectral range of $3000-3700 \mathrm{~cm}^{-1}$. The changes in water electrical conductivity under the heating by various metallic and dielectric materials are of about the same order [2-4].

The human body is believed to have their own radiation which emits into surrounding body space [5]. The existence of human radiation is identified as electromagnetic field generated by and contained within the biological system of a body [6]. The vibration of electromagnetic field generated by human body is referred as a frequency radiation of human body, which emits their radiation around the body due to its electromagnetic activities. The radiation of the human body encircles the physical body as a sphere of radiation and vibrates at their own characteristic of frequencies [7].

The experiments in [5] have shown that the characteristic of human body radiation frequency can be even used to classify the gender.

The data presented in [8] indicate that there are marked variations in the thermal properties of human skin as reported by various investigators and that the optical properties of the skin are functionally related to the water content of the skin and vary as the site of interest is changed.

Spectral radiative properties of the human body were studied experimentally in [9] in the region from the ultraviolet to the far-infrared to analyze the thermal response of the human body exposed to solar radiation and infrared radiation. Fairly large values for hemispherical reflectances are observed in the visible and near-infrared regions but very small values for hemispherical reflectances are observed in the infrared region. The absorption 
coefficient is very close to that of water and large in the infrared region [9].

Many biologists have promulgated biologic field theories to explain both biologic development and the integrity of organisms [10-12]. They suggested that an understanding of a wide variety of bio-electric phenomena in the living organism can best be reached by the assumption of an electro-dynamic field in the organism [10].

In this paper the sensors (receivers) with distillated water as a working fluid were used while investigating the biothermal and nonorganic radiation $[13,14]$. Due to the high water content of living organisms, the receiver and radiating element are highly correlated in frequencies. This can improve their sensitivity. Water reaction on various actions is of great importance for the control of vital processes including human physiological state of the operators of sophisticated equipment (aerospace, radioelectronic control systems, air traffic control services, transport etc.).

The paper is organized as follows. In Section 2 the experimental methods and measuring technique are described. In Section 3 the experimental results are presented and finally in Section 4 obtained results are analysed and some conclusions are drawn in Section 5.

\section{Experimental Methods and Technique}

Our experiments are based on the measurements of water electrical conductivity and some parameters connected with temperature coefficient of electrical conductivity as well. The main experimental procedure includes the next steps: water preparation; rinse, cell training and water pouring; stationary water heating by electric heater and electrical conductivity measurements in both cells; action on one of the cells with simultaneous thermal heating; data approximation and determination of parameters.

The water electrical conductivity was determined by conductometric method where dielectric rectangular bodies of sensors with built-in stainless steel or platinum electrodes and thermistors for water temperature control were used as measuring cells (Figures 1 and 2). The typical dimensions of receiving window were $2 \times 4 \mathrm{~cm}$; the sensor body height was $\sim 0.5-1 \mathrm{~cm}$. AC voltage with frequency of about $1 \mathrm{kHz}$ and amplitude of $\leq 1 \mathrm{~V}$ was applied as a power supply to electrodes.

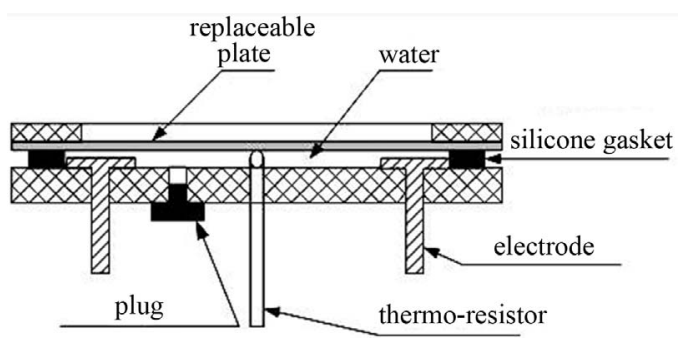

Figure 1. Conductometric sensor setup.
The signals from the circuit of electrodes and thermistor were processed by electronic module and data acquisition card. The special software ASTRA to process and visualize experimental data was developed (Figure 3).

The distillated water with initial conductivity of $2-4$ $\mu \mathrm{S} / \mathrm{cm}$ was used. The impact on water by infrared and $\mathrm{THz}$ electromagnetic radiation was made by heaters from various materials and by biological objects as well. The copper carbonized plate heated by nichrome wire up to $40^{\circ} \mathrm{C}$ was used as a solid irradiator (heater).

To determine the water reaction on emission from different mediums the thin foils made from various materials and fixed alternately at the surface of the plate were used. The foils were heated for $10 \mathrm{~min}$. and then were placed at the distance of about $2 \mathrm{~mm}$ above the water surface. Two identical sensors one of which was a control sensor were used. One can define two different cases in experimental procedure of determination of irradiation action of solid-state foils:

1) Water heating in control sensor by a standard thermal heater close to the black body irradiator and;

2) Water heating in measuring sensor by various heated foils.

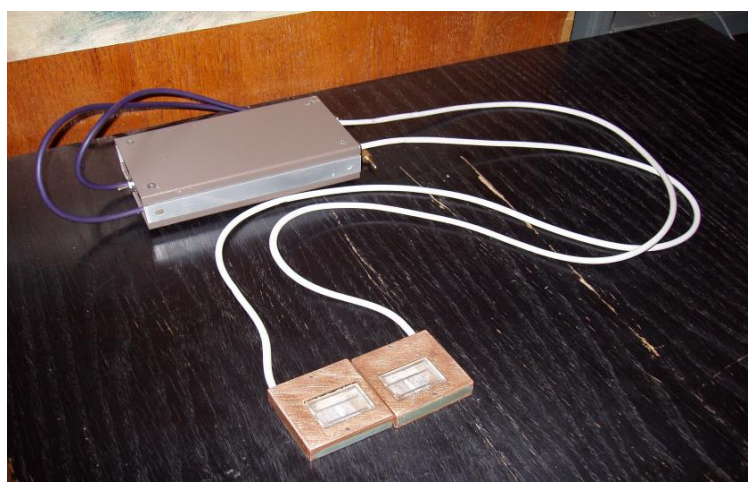

Figure 2. Measuring and control sensors along with electronic module.

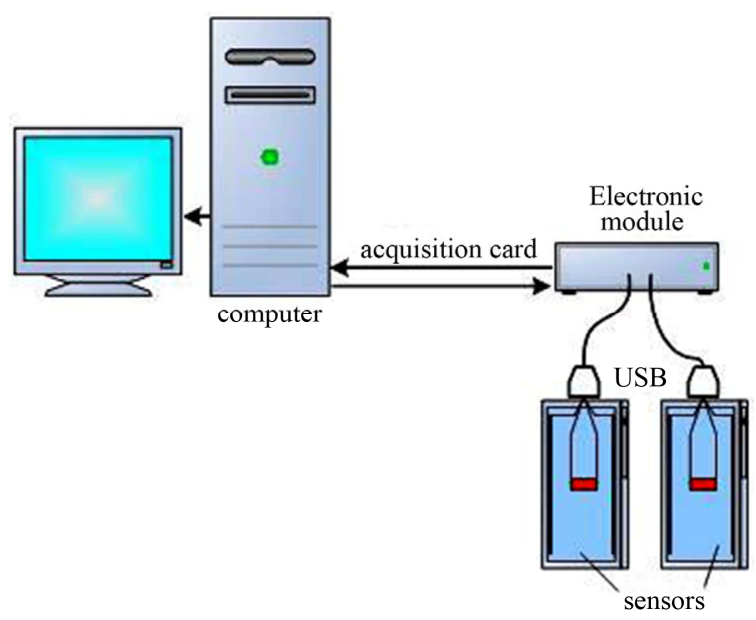

Figure 3. Experimental setup. 
The effect was determined by the difference of these two sensor readings.

Besides above mentioned experimental technique for determining the level of action of heated water on water in conductometer we used sensors and elements with somewhat different technical specifications [3].

The electrical conductivity was measured in the temperature range of $23^{\circ} \mathrm{C}-28^{\circ} \mathrm{C}$. In the first case the electric heater 1 served as a heat source for water in volume $\mathrm{V}_{1}$ (Figure 4(a)). In the second case the water vapors, water thermal emission and vessel walls served as a heat source (Figure 4(b)). Water in the volume 2 in its turn was heated by electric heater 1 . When using water vapors as a heat source the stronger dependence of electrical conductivity on temperature at all examined ranges was observed in comparison with the heating by electrical heater. This is in a close agreement with results obtained in [4].

To measure quantitatively the observed deviation two parameters were used in addition to the direct measurement of electrical conductivity. The first one is determined as

$$
a=\frac{1}{\sigma} \frac{\partial \sigma}{\partial T}
$$

where $\sigma$ is an electric conductivity and $T$ is a temperature. The parameter $a$ is a relative temperature coefficient of electrical conductivity and it describes the water properties. The difference due to heating methods can be described by the following parameter

$$
B=\frac{\Delta \sigma_{B}-\Delta \sigma_{T}}{\sigma_{0}} \frac{1}{\Delta T}
$$

where $\Delta \sigma_{B}$ is an electrical conductivity increment during the action of a biological object (bioaction), $\Delta \sigma$ is an electrical conductivity increment during the usual heating, $\Delta T$ is a period of bioaction/heating and $\sigma_{0}$ is an initial value of electric conductivity.

Nominally parameter $B$ can be viewed as a bioaction power (a relative excess of an electrical conductivity

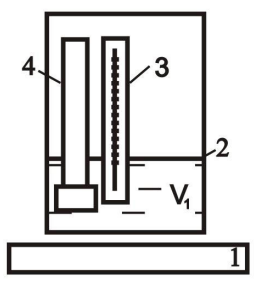

a

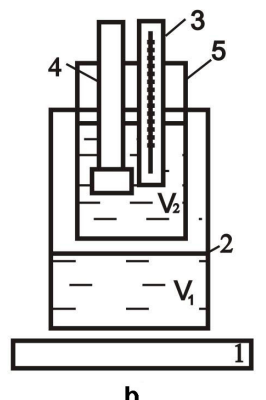

Figure 4. Experimental setup for water electrical conductivity measurements. Here 1-electric heater, 2-water volume, 3-thermometer, 4-conductometer, 5-examined water vessel. increment during bioaction over an electrical conductivity increment during traditional heating). The use of these two parameters allows to increase the sensitivity and accuracy of results obtained. In our experiments the rate of temperature increase was about $1^{\circ} \mathrm{C} / \mathrm{min}$. As a rule, the impact of biological object on water was made by water irradiation by central part of experimenter's palm located $2 \mathrm{~mm}$ above the water surface.

\section{Results}

The results presented in Figure 5 are the generalization of a great number of experiments. In the obtained dependencies of electrical conductivity on radiation $\sigma=f(T)$ one can single out 4 curve classes. In the first class the dependence $\sigma=f(T)$ is approximately linear in the temperature range of $22^{\circ} \mathrm{C}-28^{\circ} \mathrm{C}$ and the rate of change is $\sim 2.2 \%-3 \% /{ }^{\circ} \mathrm{C}$. This is close to standard reference data. This class consists of the irradiators made of copper, lavsan, fiberglass and cellulose triacetate. In the second class (lead, magnesium, cellophane) the change of $\sigma=f(T)$ is mainly focused around $3.5 \%-4 \% /{ }^{\circ} \mathrm{C}$. The exposure of water in a measuring sensor to radiation of water heated in glass vessel [3], transparent to the radiation of wavelength less than $4 \mu \mathrm{m}$ (determined by pass band of water vessel) and of thin water films on the surface of various materials including $50-60 \mu \mathrm{m}$ water films on operator hand can be put into the third class where the change of conductivity was $\sim 6 \%-8 \% /{ }^{\circ} \mathrm{C}$. The fourth class is formed by curves of electrical conductivity

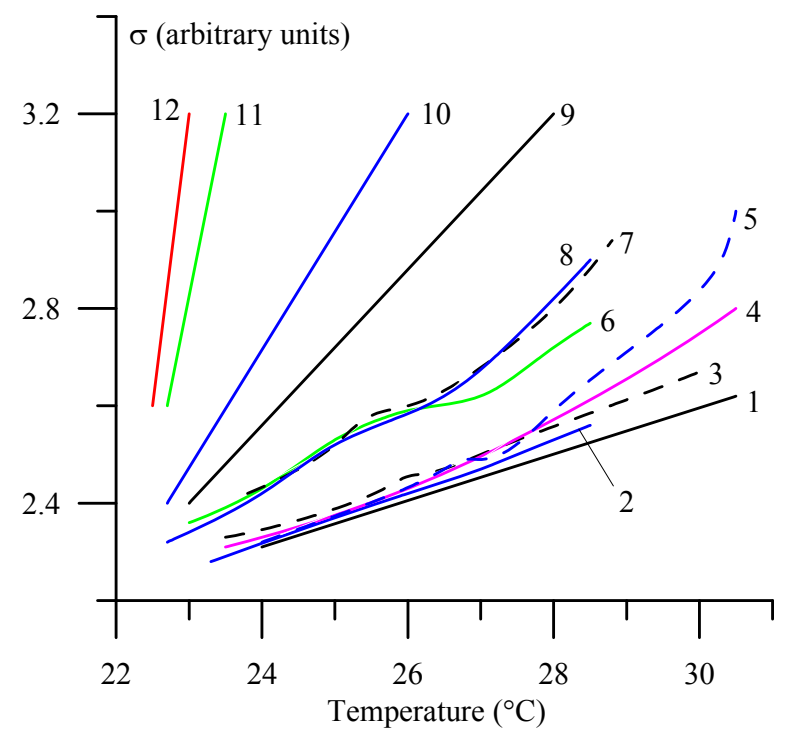

Figure 5. Water electrical conductivity versus temperature for various materials: 1 -black body, 2 - copper, 3 -lavsan, 4-fiberglass, 5-cellulose triacetate, 6-lead, 7-magnesium, 8-cellophane, 9-water in glass vessel, 10 -water 50 - $60 \mu \mathrm{m}$ film on the surface of operator palm, 11-operator palm, 12-operator palm dried by talc. 
and conductivity temperature coefficient $(1 / \sigma)(\partial \sigma / \partial T)$ obtained during water heating by experimenter's palm when the rate of change was about $20 \%-50 \% /{ }^{\circ} \mathrm{C}$.

The intensity of bioaction on water from operator palm is illustrated by Figure 6 where the dependence of specific conductivity $s$ on temperature is shown. It can be seen that the change of specific electrical conductivity for water heating up to $24.7^{\circ} \mathrm{C}$ by electrical heater is the same for control and measuring sensors. At that temperature the operator action on the water in measuring sensors starts and the rate of change of conductivity increases abruptly (curve 1). In the control sensor for continued heating by electrical heater the curve slope is not changed (curve 2).

The experimental studies of bioaction propagation through various materials have shown that most of metallic, dielectric and polymer foils strongly attenuate this radiation. However a number of materials have special permeabilities.

The nonmonotonic change of absorption for the increase in the thickness of absorption material $(A I)$ is observed as well. In these measurements the $A l$ foil with thickness of $0.005 \mathrm{~mm}$ was used. When the number of foil layers was changed the periodical radiation intensity differences (similar to interference distribution of light intensity) 2.3 - 8 times larger were observed (Figure 7).

The intensity of suprathermal radiation $B$ is an individual parameter specific for each human. But this is true only for an average value of this parameter. Its current value is subject to variation over wide range $( \pm 50 \%)$. As was determined, the changes of intensity of suprathermal radiation depend on psycho-emotional human state considerably.

The inference about higher nervous activity influence on parameters of palm radiation was made when the following facts had been elicited. Parameter $B$ measured by

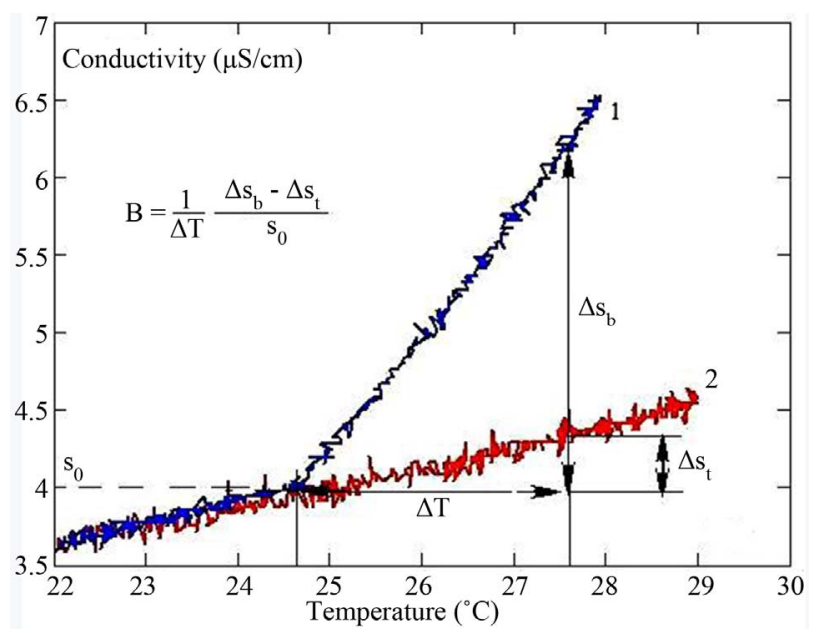

Figure 6. The dependence $\sigma=f(T)$ for the water heating by palm (1) and by electrical heater (2).

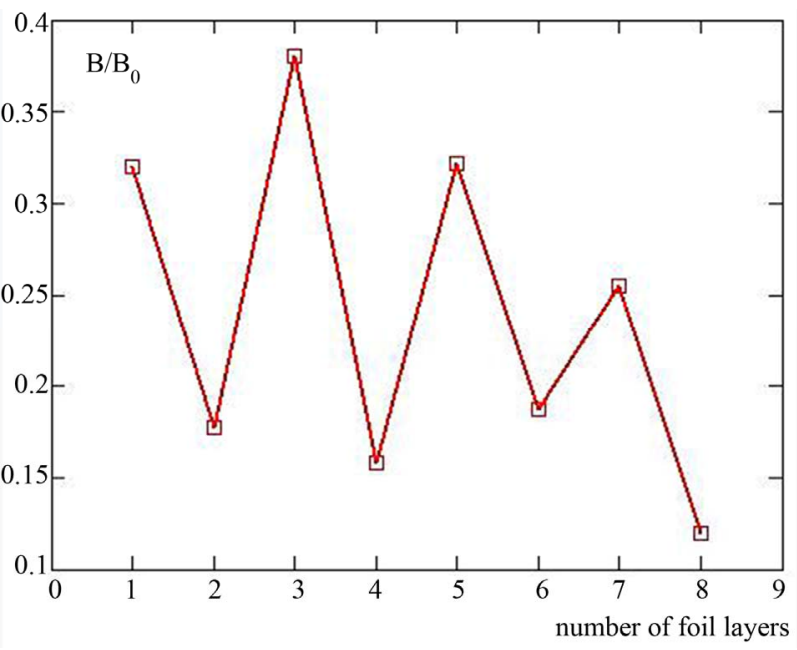

Figure 7. Dependence of bioradiation on different numbers of $A l$ foil layers.

water sensor decreased monotonous so far as the test person experienced growing up fatigue. Besides, the substantial variations of $B$ are observed for changes of mood of test persons. The measurements performed for test persons in normal states allowed to obtain statistical distribution of $B$ values.

As a part of experimental study, the asymmetry in the distribution of radiation intensity for water heating by the left and the right palms was checked out. The results obtained are shown in Figure 8. It can be seen that the bioradiation is not symmetrical relative to the left and the right part of human body. It should be noted that the actions of left and right hand on the water in the sensor differ in magnitude. As a rule for men the parameter value for the right hand is larger than for the left hand. The reverse holds true for women. However the current values depend on the test person state and following the changes of the latter the inversion can be observed. Moreover the results are individually specific.

It can be noted that the curves in Figure 8 are approxi-mation of the distribution histograms of $B$ for the right and left hands of test persons. The points on $X$-axis correspond to values of $B$ and the points of $Y$-axis correspond to the ratio of test person number with given value of B to the total number of test persons. A total of 53 students were examined. While analyzing the results the total range of $B$ values was divided into 5 - 20 segments with varying length. This led to the changes in the look of the curve but in all cases there were two maxima. It is the evidence that there are either two groups of people distinguished by the power of bioaction or two stable human states with different bioaction powers. Distribution curve spreading-out relative to its maximum values can be connected with the dependence of bioaction power on test person state, on his response to changes in 


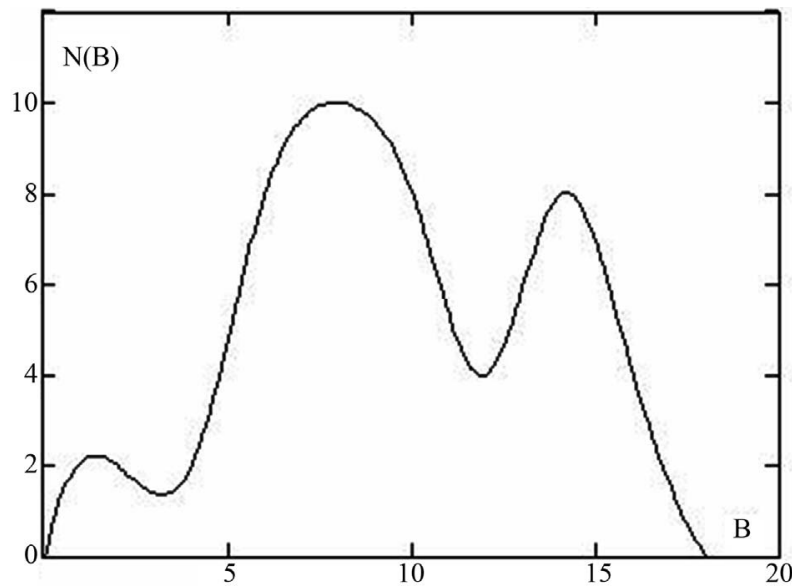

(a)

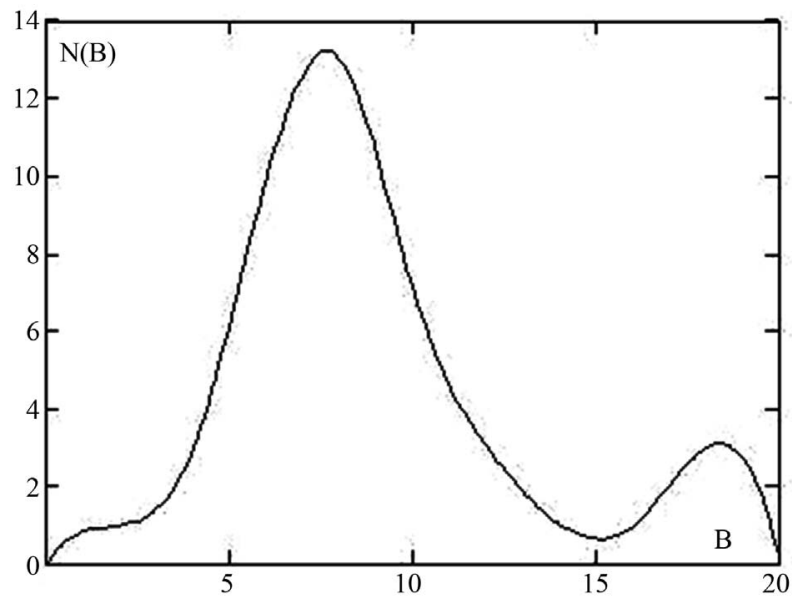

(b)

Figure 8. Distribution of radiation intensity for water heating by the right (a) and the left (b) palms.

outward conditions and on other factors of random nature. However the range of such changes is not that substantial to smooth the double-peaked distribution curve.

The mentioned earlier tendency towards the decrease of parameter $B$ values for fatigue states was scrutinized for the group consisting of 39 students while they were fulfilling the task by PC with duration of 3 - 4 hours. The measurements were carried out before and immediately after the task fulfillment. The results obtained are presented in Figure 9. For illustration purposes the histograms of above mentioned experiment (fatigue-solid line, arousal-dashed line) are smoothed by splines. It can be seen that the fatigue and emotional arousal lead to opposite results relative to parameter $B$ changes.

Another series of measurements was carried out to obtain and analyze the data for emotional arousal different from reported above. Each test person had to carefully watch the purely decorative picture on PC display. From time to time unpleasant image unexpectedly appeared on the display accompanied by a harsh sound. It led to a

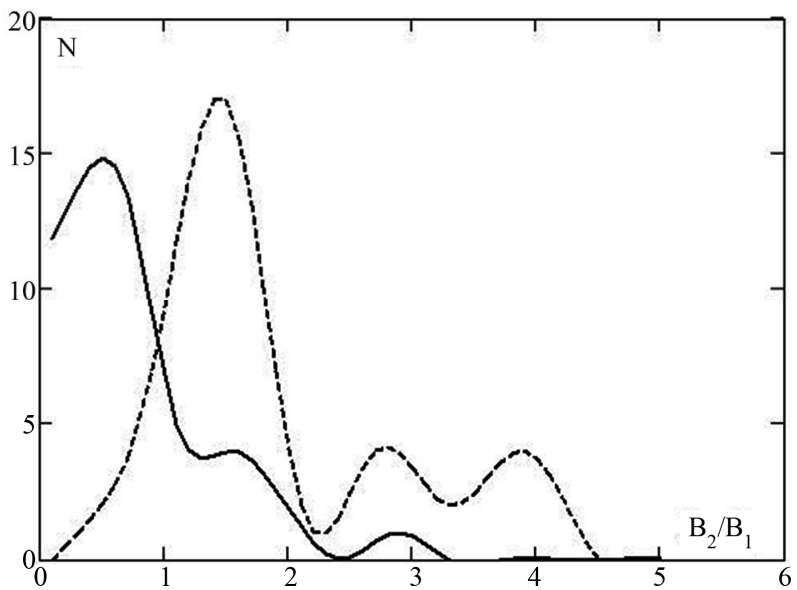

Figure 9. The dependencies of ratio $B_{2} / B_{1} . B_{1}$ are values before emotional influence, $B_{2}$ are values after influence. Fatigue-solid line, arousal - dashed line.

startle response of a person. The biometric parameters of test persons were measured before and after this response. The distributions similar to those in Figure 9 were obtained. Besides, such distributions were obtained while analyzing the experimental data corresponding to the measurements performed before and after student's exams and in some other cases.

\section{Discussion}

Experiments performed so far do not allow determining unambiguously the physical nature of discovered suprathermal radiation of humans. As a hypothesis one can suggest that besides the usual changes in electrical conductivity of water during its heating there is some mechanism connected with the resonant absorption of radiation in the infrared or submillimeter spectrum range (Figures 5 and 7).

To determine the nature of anomalous radiation acting on the water, the experiments measuring the spatial distribution of radiation intensity were carried out. The sensor was moved through the dielectric or metal waveguide and the operator palm acted upon the open end of the waveguide. It led to the changes in sensor readings relative to the distance $h$ between the operator palm and water level.

It only remains to suggest that electromagnetic waves forming a constituent part of bioradiation lie in the shorter length range where the geometrical optics approximation can be applied for the description of their propagation. Assuming the isotropy of biological object radiation and neglecting for the sake of simplicity the reflection from waveguide walls, one can obtain the expression describing the dependence of power $W$ of emission falling to the sensor on its distance from the radiator $h$ (experimenter's palm located at the waveguide cut off in this case): 


$$
\begin{aligned}
W(h)= & \left(2 a \cdot \operatorname{arctg}(a / h)-h \cdot \ln \left(1+a^{2} / h^{2}\right)\right) \times \\
& \times\left(2 b \cdot \operatorname{arctg}(b / h)-h \cdot \ln \left(1+b^{2} / h^{2}\right)\right)
\end{aligned}
$$

where $a$ and $b$ are dimensions of wide and narrow waveguide walls.

In Figure 10 the dependence of (normalized to its initial value) parameter characterizing the intensity of suprathermal radiation on the distance $h$ for metal waveguide (crosses) and dielectric waveguide (circles) is shown as well. The close agreement of experimental and theoretical data give evidence to the suggestion that suprathermal radiation is electromagnetic one with wave length much less than $15 \mathrm{~mm}$ that is determined by the sizes of waveguide. The difference in the locations of experimental points corresponding to metal and dielectric waveguides is explained by the difference in reflection coefficients of radiation for metal and dielectric walls not accounted in deriving the Equation (3).

Let us turn to the analysis of Figure 5. There the experimental data (curves $2-8$ ) refer to the water exposure to the heating by nonbiological materials. Curves $9-12$ refer to water heating by water and/or biological objects. The differences in water reaction to the heating by various nonbiological materials are coming probably from their emissivity peculiarities and emission spectrum [1]. Curves 9 - 12 refer to the case where the irradiator (water, water films, operator hands containing large amount of water) and receiver (water) have a common inherent element viz. water. Since the spectral features of receiver and irradiator agree to a large extent, their interaction can be of resonant nature.

The conductivity in sensors changed mostly due to two factors: radiation as a physical factor and influence of chemical substances excreted by experimenter's tissue

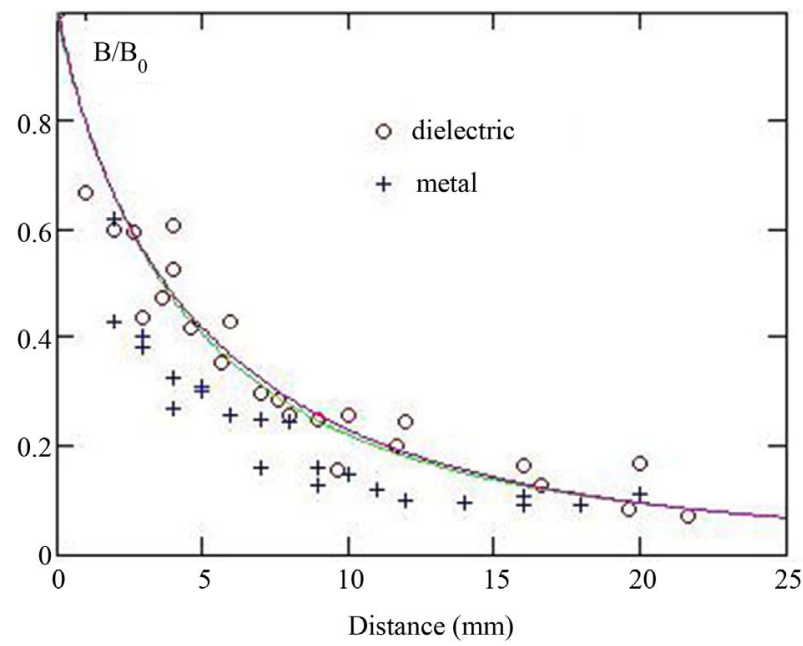

Figure 10. Dependence of normalized to its initial value parameter characterizing the intensity of suprathermal radiation on the distance between the human palm and water level for metal and dielectric waveguides. and skin, for example, $\mathrm{CO}_{2}$ and sweat. The special experiments carried out with the purpose of revealing the role of these factors in $\sigma=f(T)$ dependence showed that their ratio can change substantially. Some results of these experiments are illustrated in Figure 5 by curve 10 that refers to the case when the part of the hand irradiating the receiving section of the sensor was covered by 50 - $60 \mu \mathrm{m}$ water layer. The hand surface was carefully dried and deoiled beforehand. The water layer covered sweat pores completely thereby preventing perspiration and $\mathrm{CO}_{2}$ excretion. Infrared, $\mathrm{THz}$ and microwave radiation partly passed, partly was absorbed by water layer and heated it as well. As a result the water in sensor was under the influence of both direct hand emission and reemission of water layer on hand surface. In this case the changes in electrical conductivity are greater than for the irradiation by water in glass vessel (curve 9) when the biological object component is absent.

The absorbed radiation energy is expended not on the temperature increase but directly influences on water transport properties, e.g. it changes the water structure and the structures of hydration shell of impurities. Such radiation can be generated by the luminescence at the one of numerous chemical reactions in cells of living organism. Just as well one can assume that human body radiation is of equilibrium nature and its spectrum coincides with the spectrum of black (or grey) body with temperature $36^{\circ} \mathrm{C}-37^{\circ} \mathrm{C}$. However, passing through the skin it is weakened in the wide spectral range except for some narrow pass band close to the band of water resonant absorption [15]. In any of these cases the excess of values of water electrical conductivity over its equilibrium value for given temperature will be observed.

Completely different results are obtained when the hand is carefully dried by talc. As a matter of fact the talc blocks up the pores preventing sweet excretion and removes the water film on the skin surface as well. Accordingly the thermal emission and $\mathrm{CO}_{2}$ reaches the sensor and that leads to the substantial changes in water conductivity (curve 12). This is confirmed by curve 11 obtained for hand radiation in usual conditions.

Thus analysis done has shown that the phenomena mechanisms could be connected with the excitation of vibration-rotation levels of water molecules under the influence of radiation where intensive absorption lines exist. The molecule excitation leads to the change in dissociation energy and in water polarization (due to the increase in molecule sizes during the excitation of vibration-rotation levels [16]). As a result water electrical conductivity becomes larger due to the increase both in charged particle concentration (owing to the decrease in dissociation energy for the excitation to the vibrationrotation levels) and in the polarization leading to the water structuring and hence to the gain in particle mobility- 
ites. This is also confirmed by NMR investigations showing that the water molecular structure is greatly changed under the influence of long wave infrared emission. This leads to the substantial increase in molecular mobility [13] and, hence to the electrical conductivity changes. Besides as is shown in [4], the decrease of water entropy and the increase of its conductivity are observed during water heating by biological object.

\section{Conclusions}

Thus one can conclude that $\sigma=f(T)$ rate of change is higher for biothermal radiation compared with the thermal radiation of other materials and is dependent on the state of human. The results presented reveal the role of water in radiation processes and measurement procedures. However a lot of basic biological and biophysical questions relevant to bioradiations are still to be further explored and answered.

The nature of observed phenomena is extremely complicated. However the analysis done have shown that the phenomena mechanisms can be connected with the excitation of vibration-rotation levels of water molecules under the influence of radiation where intensive absorption lines exist.

The results obtained have good perspectives for future applications in medical diagnostics and especially in the status control of operators of complex systems in aviation and space techniques, radioelectronic control systems, air traffic control services, information and telecommunication systems.

\section{REFERENCES}

[1] L. N. Latyev, V. A. Petrov, V. E. Chekhovsky and E. M. Shestakov, "Radiant Properties of Solid Bodies, Reference Book," Energy, Moscow, 1971.

[2] A. T. Lukyanov, "Correcting the Notion of Temperature of Avogadro law," Proceedings of International Conference "Recent Developments in Applied Mathematics and Mechanics: Theory, Experiment and Practice", Novosibirsk, 24-29 June 2001, pp. 270-272.

[3] G. G. Shishkin, V. G. Rebrov, A. D. Usanov and D. A. Usanov, "Influence of Heating Source on Water Electrical Conductivity and on Infrared Spectrum of Absorption," Biomedical Radioelectronics, No. 11, 2011, pp. 56-60.

[4] I. M. Ageev and G. G. Shishkin, "Change in the Electrical Conductivity of Water on Heating by Various Heat Sources Including Biological Objects," Biophysics, Vol.
47, No. 5, 2002, pp. 726-730.

[5] S. A. Jalil, M. N. Taib, H. Abdullah and M. M. Yunus, "Frequency Radiation Characteristic around the Human Body," International Journal of Simulation: Systems, Science \& Technology, Vol. 12, No. 1, 2011, pp. 34-39.

[6] K. J. Hintz, G. L. Yount, I. Kadar, G. Schwartz, R. Hammerschlag and S. Lin, "Bioenergy Definitions and Research Guidelines," Alternative Therapies in Health and Medicine, Vol. 9, 2003, pp. 13-30.

[7] A. R. Liboff, "Toward an Electromagnetic Paradigm for Biology and Medicine," Journal of Alternative and Complementary Medicine, Vol. 10, No. 1, 2004, pp. 41-47. doi:10.1089/107555304322848940.

[8] M. L. Cohen, "Measurement of the Thermal Properties of Human Skin. A review," Journal of Investigative Dermatology, Vol. 69, No. 3, 1977, pp. 333-338. doi:10.1111/1523-1747.ep12507965

[9] N. Terada, K. Ohnishi, M. Kobayashi and T. Kunitomo, "Spectral Radiative Properties of a Living Human Body," International Journal of Thermophysics, Vol. 7, No. 5, 1986, pp. 1101-1113. doi:10.1007/BF00502381

[10] H. S. Burr and F. S. C Northrup, "Evidence for the Existence of an Electro-dynamic Field in Living Organisms," Proceedings of the National Academy of Sciences of the United States of America, Vol. 25, No. 6, 1939, pp. 284-288. doi:10.1073/pnas.25.6.284

[11] M. Bischof, "The History of Bioelectromagnetism," In: M. W. Ho, F. A. Popp and U. Warnke, Eds., Bioelectrodynamics and Biocommunication, World Scientific Publishing, Singapore City, 1994, pp. 1-30.

[12] B. Rubik, "The Biofield Hypothesis: Its Biophysical Basis and Role in Medicine," Journal of Alternative and Complementary Medicine, Vol. 8, No. 6, 2002, pp. 703717. doi:10.1089/10755530260511711

[13] K. Matsushita, "Evolution of the State of Water by NMR Spectrometry," FIR Joho, Vol. 5, 1988, pp. 6-10.

[14] G. G. Shishkin, I. M. Ageev and A. G. Shishkin, "Measurements of Non-Equilibrium Thermal and Biothermal Radiation by Water Conductometric Sensors," Proceedings of the 37th International Conference on Infrared, Millimeter and Terahertz Waves, Wollongong, 23 - 28 September 2012, pp. 1-3. doi:10.1109/IRMMW-THz.2012.6380166

[15] J. D. Hardy and C. Muschenheim, "The Radiation of Heat from the Human Body IV. The Emission, Reflection, and Transmission of Infra-red Radiation by Human Skin," The Journal of Clinical Investigation, Vol. 13, No. 4, 1934, pp. 615-620. doi:10.1172/JCI100609

[16] G. N. Zatsepina, "Physical Properties and the Structure of Water," Moscow University Publishing, Moscow, 1998. 